\title{
ASPECTOS LÓGICOS DE LA INTUICIÓN: APROXIMACIÓN AL ANÁLISIS DE UNA DECISIÓN ECONÓMICA EN UN CONTEXTO DE GLOBALIZACIÓN
}

\author{
Horacio Manrique Tinés \\ Isabella Builes Roldán \\ Escuela de Humanidades - Universidad EAFIT, Medellín-Colombia
}

\begin{abstract}
https://doi.org/10.33676/EMUI_nomads.55.08
Resumen: El objetivo de este artículo es mostrar la utilidad que puede representar el análisis de una decisión en el que se tengan en cuenta los aspectos lógicos de la intuición. Para ello, en un primer momento, definimos qué entendemos por decisión y por intuición, y qué identificamos como sus aspectos lógicos. Luego, presentamos un caso de toma de decisiones de un vicepresidente de una empresa de inversión en bolsa de valores, ubicando algunos aspectos importantes en relación con la globalización, tema central de estas jornadas. A continuación, resaltamos los elementos lógico-intuitivos que aparecen en el proceso decisorio del caso analizado. Finalmente, argumentamos qué papel cumple la intuición en este caso de toma de decisiones económicas en un contexto de globalización y qué implicaciones tiene esta propuesta de análisis sobre las decisiones existenciales que, en última instancia, puede terminar siendo cada una de las decisiones que tomamos.
\end{abstract}

Palabras clave: Lógica, intuición, toma de decisiones, decisiones económicas, decisiones existenciales.

\section{Logical aspects of intuition: Approximation to the analysis of an economic decision in a context of globalization}

Abstract.- The objective of this article is to show the usefulness of the analysis of a decision that takes into account the logical aspects of intuition. To do that, at a first moment, we define what we understand by decision and intuition, and what we identify as its logical aspects. Then, we present a case of decision making of a vice president of an investment company in stock market, placing some important aspects in relation to globalization, the central theme of these days. Next, we highlight the logical-intuitive elements that appear in the decision-making process of the analyzed case. Finally, we argue what role intuition plays in this case of economic decision making in a context of globalization and what implications this proposal of analysis has on the existential decisions that, in the end, can end up being each of the decisions we make.

Keywords: Logic, infuition, decision making, economic decisions, existential decisions. 


\section{Introducción}

Definiremos la decisión como un "juicio resolutorio (síntesis) sobre una acción que el agente tiene la posibilidad presente o futura de realizar, producto de un análisis (consciente o inconsciente)" (Autor, 2017, p. 10). Dado que la decisión es un juicio, es un producto mental, por lo que no implica necesariamente la realización de la acción, a la que llamamos elección (Lopera, Echeverri y Goenaga, en prensa; Autor, 2017).

Además, la decisión en tanto juicio resolutorio o conclusión práctica ('haré aquello' o 'haré esto'), está fundamentada en una serie de juicios previos o pre-juicios (Ramírez, Lopera, Zuluaga y Ortiz, 2017; Lopera, Ramírez, Zuluaga y Ortiz, 2010), que son creencias o valores que hemos ido incorporando a lo largo de la vida sin cuestionarlos y que funcionan como premisas, muchas veces de forma no consciente. Desde nuestra perspectiva, los prejuicios pueden ser identificados enjuiciados, cuestionados, con el fin de captar si efectivamente estamos de acuerdo con ellos, y en caso negativo, cambiarlos o modificarlos, de manera que operen como pre-supuestos, esto es supuestos o premisas de las cuales partimos para tomar las decisiones, pero flexibilizándolas de manera que posibiliten una apertura y una escucha de la situación (Ramírez et al, 2017).

Desde la perspectiva de la psicología cognitiva, se diferencian dos modos de pensamiento denominados proceso 1 (o intuición) y proceso 2 (o deliberación). El proceso uno se caracteriza por ser rápido, automático, sin esfuerzo y emocional. El proceso 2, se caracteriza por ser lento, controlado, que requiere esfuerzo y emocionalmente neutro (Kahneman, 2012).

Desde nuestra perspectiva, tal caracterización de los dos procesos, deja de lado los aspectos centrales de los mismos, que son los aspectos lógicos que subyacen a cada modo de pensar. Por lo tanto, entenderemos la intuición desde tres perspectivas relacionadas: como una facultad que implica captar una gran cantidad de datos o indicios sin tener que enumerarlos conscientemente; mediante un proceso de pensamiento (intuir), no consciente, y automático, resultado de articulaciones inconscientes; es decir, un proceso de análisis inconsciente en el que no interviene la deliberación, pero que permite establecer asociaciones entre los indicios captados. Este proceso genera, usualmente de forma rápida, una serie de resultados que llamamos intuiciones y que se manifiestan como sensaciones, estados de ánimo, presentimientos y ocurrencias (Ramírez et al., 2017; SadlerSmith, 2016). Las intuiciones, como resultado del intuir, tienen como aspecto destacable su rapidez en cuanto a su aparición en la conciencia. Las intuiciones permiten generar respuestas adecuadas en muchas situaciones, pero también tienen como característica la cualidad de 'verdad' o certidumbre, que puede llevar al dogmatismo a 
quien la experimenta. Esto se explica por estar basadas frecuentemente en prejuicios, que pueden conducir a sesgos errores en la decisión o en la elección. Nuestra propuesta es que es posible formar la intuición, es decir, que luego de un proceso de ascesis subjetiva que implica el desprejuiciamiento (Ramírez et al, 2017; Lopera et al., 2017), el proceso de pensamiento intuitivo será más flexible y fluido, al estar basado cada vez más en presupuestos (no en prejuicios), y así, puede llevar a respuestas más válidas y convenientes de acuerdo con cada situación.

Ahora, sostenemos la hipótesis de que el pensamiento intuitivo posee una lógica (o aspectos lógicos) subyacente a este, y optamos por comprenderlo desde estas categorías lógicas, más allá de una perspectiva fenoménica. En otras palabras, el intuir, además de poseer características como ser un proceso generalmente emocional, holístico, no consciente y rápido (Dane y Pratt, 2007), puede comprenderse como un modo de procesamiento psíquico que combina dos tipos de lógica: la lógica primaria y la lógica secundaria, las cuales interactúan entre sí conformando una lógica fractal (Ramírez, 2012; Autor y Autor, 2018). En términos generales, la lógica primaria está basada en la contigüidad y la semejanza (metonimia y metáfora); y la lógica secundaria en el principio de no contradicción y bivalencia. La lógica fractal implica una mezcla o una alternancia entre ambos tipos de lógica.

En el ser humano, tanto la lógica primaria como la lógica secundaria tienen un fundamento biológico y fisiológico, por ejemplo, en cuanto al funcionamiento neural y la comunicación entre neuronas mediante la descarga o inhibición de neurotransmisores (Damasio, 2006). También, la intuición se da gracias a procesos fisiológicos y significativos como, por ejemplo, los marcadores somáticos (Damasio, 2006), entendidos como marcas en el cuerpo que dejan los significados de las experiencias vividas.

La lógica primaria se asocia fundamentalmente a las leyes del proceso primario descritas por el psicoanálisis y que están guiadas por la consecución del placer y la evitación del displacer a corto plazo (principio del placer) (similar a la aversión a la pérdida de la que habla Kahneman, 2012); también se asocia al pensamiento basado en imágenes, como el utilizado en la imaginación creafiva, el arte o los mitos (Lévi-Strauss, 1995). El proceso primario, se refiere a una forma de pensamiento en la cual se privilegian las imágenes en vez de los conceptos (lo imaginario en vez de lo simbólico); no permite diferenciar entre la realidad y la fantasía. En la lógica primaria las representaciones se asocian mediante dos categorías básicas: la semejanza y la contigüidad (lo que Freud llama condensación y desplazamiento, Lacan metáfora y metonimia, y que desde la psicología de la Gestalt se denomina principio de similitud, por un lado, y principio de proximidad, por otro). 
La lógica secundaria se asocia con el pensamiento simbólico, reflexivo, deliberado, crítico, basado en los principios de no contradicción y bivalencia, que se evidencian frecuentemente en aquellas tareas que requieren un esfuerzo cognitivo controlado conscientemente y que se desarrollan lentamente (el sistema 2 que retoma Kahneman, 2012). Desde el psicoanálisis, se hablaría aquí del principio de realidad, que contribuye a la realización de metas a largo plazo que, aunque en principio el proceso requiera mayor esfuerzo, se estima que a largo plazo será más beneficioso.

En la vida cotidiana, estos dos tipos de lógica no son excluyentes entre sí, de hecho son complementarios; por eso la lógica fractal es una combinación de los dos tipos de lógica anteriores y es la lógica que utilizamos cotidianamente (Ramírez, 2012). Usualmente nos basamos en un pensamiento que mezcla, alternando, ambas lógicas, pero de manera automática y no consciente. Así, pues, la mayor parte del tiempo operamos de forma intuitiva (Gigerenzer, 2008), sin darnos cuenta de los procesos que llevamos a cabo a la hora de tomar una decisión o hacer alguna elección.

\section{Presentación del caso}

De acuerdo con los conceptos expuestos hasta el momento, lo que proponemos en este escrito es analizar hasta cierto punto un caso de toma de decisiones de un vicepresidente de una compañía de inversiones en la bolsa de valores, identificando aquellos aspectos lógicos que intervinieron en el proceso que le permitió llegar a una decisión.

Presentamos entonces el caso de un vicepresidente de una compañía financiera ${ }^{1}$ que narra una situación en la cual él, que en ese momento trabajaba en un banco, tomó la decisión de comprar acciones de otro banco [se omite el nombre del banco] "y desafortunadamente la acción cayó de 60 dólares a 90 centavos, o sea, se perdió el $99 \%$ de la plata. Esa decisión de haber tomado ese riesgo fue mal medida. O sea, lo que estoy diciendo es que el escenario peor, siempre el escenario peor fue considerado como la pérdida como del 30 o 40\% del capital, nunca el $100 \%$ del capital; y aquí se perdió el $100 \%$ del capital".

Al evaluar la situación considera que "hubo un exceso de delegación a una persona que no fue cuidadosa en la información. Y, de hecho,

\footnotetext{
1 Este caso se extrajo de una entrevista realizada en el marco de la tesis de doctorado del primer autor que aborda el proceso de pensamiento deliberado e intuitivo en la toma de decisiones de directivos organizacionales. La entrevista contó con la firma del consentimiento informado del participante y aquí se presenta de forma general y anónima, siguiendo los estándares éticos exigidos para la investigación con seres humanos en la Declaración de Helsinki.
} 
cuando pedí la información, lo que él me pasa por correo no correspondía a lo que estaba escrito en el contrato. Pero es culpa mía por no haber leído el contrato, no fue culpa de él".

Cuando analiza los motivos, o prejuicios, que lo llevaron a tomar esa decisión, afirma: "Delegué en exceso. Delegué en exceso en esa persona y nunca profundicé. ¿¿Sí me entiende? Simplemente pregunté: 'muéstreme cuál es el piso de esta nota estructurada', él me dice que es, creo que a 60 ० 70\%; es decir, la pérdida máxima era como del 30\%, la utilidad podía ser mucha; pero cuando se ejecuta él viene, inclusive sudando, y me dice que leyó mal. Y ya no había nada que hacer, la acción... ya [...] estaba prácticamente en 90 centavos. Y se perdió toda la plata".

En ese momento, dice, "sentí mucha rabia, mucha impotencia, me sentí muy má conmigo mismo por no haber profundizado en eso, no haber leído el contrato y haber delegado en exceso a una persona que era muy joven en ese momento".

A partir de esta experiencia, afirma, aprendió a "no delegar con tanta tranquilidad en las situaciones tan delicadas como [las relacionadas con] la plata. Había que revisar muy bien ese contrato y darle un doble chequeo".

\section{Aproximación al análisis del caso}

Hasta aquí el caso. Ahora bien, para comprender mejor el contexto en el que se presenta la decisión, caracterizaremos la globalización como un fenómeno generado por el amplio desarrollo de las tecnologías, especialmente las relacionadas con los medios de comunicación y de transporte. Este desarrollo posibilita que el tiempo y el espacio se compriman (aspecto ontológico, según Malvezzi, 2017, p. 37): "eventos distantes interactú[a]n como si estuviesen físicamente próximos" y "diversos aspectos de la realidad [se transforman] en información para ser manejados como tal" (Malvezzi, 2017, p. 37). Como efecto surgen el aspecto económico de la globalización, que se refiere a la "mayor facilidad para la inserción de capital financiero, capifal intelectual y tecnología en los negocios, hecho que altera la organización gerencial y la competitividad" (Malvezzi, 2017, p. 37); el aspecto antropológico, que consiste en "la rápida circulación de objetos y sujetos como consecuencia del continuo bombardeo de significantes a los eventos, lo que torna el capital simbólico en uno de los factores más relevantes en la formación de valor y significado de las personas y objetos en la sociedad actual" (Malvezzi, 2017, p. 37); y, finalmente, el aspecto psicológico, según el cual se tiene "la apertura, para que las personas experimenten distintas y variadas identidades y no estén limitadas a las 
identidades propuestas por un grupo cultural en particular" (Malvezzi, 2017, p. 37).

Un ejemplo nos lo da el entrevistado cuando plantea que "en bolsa se da que todo es de segundos. Tres minutos en bolsa es una eternidad. En bolsa la emocionalidad [implica que] 'hay que tomar la decisión ya, en segundos', y no es que se tome inconscientemente, por eso yo digo no es no consciente sino que es muy emocional". Agrega que la decisión se toma "en milésimas de segundo". Resaltamos, entonces, dos aspectos fundamentales asociados a la toma de decisiones en el contexto de la globalización: la velocidad que demanda la toma de decisiones y la emocionalidad asociada.

En este contexto de globalización, desde el aspecto ontológico, las decisiones deben tomarse muy rápidamente en una situación como la narrada; además, el caso muestra que disponer de la información no depende únicamente de la tecnología, sino de los sujetos que la interpretan; y, por estos dos aspectos que señalamos, el pensamiento intuitivo se vuelve fundamental, pues siempre hemos de contar con la participación del sujeto. Desde el aspecto económico, en el caso narrado, la información financiera, aunque distorsionada, llega rápidamente al decisor, alimentada por una intuición sobre la alta probabilidad de obtener unas utilidades y una percepción de seguridad frente a una baja probabilidad de pérdida, que genera como reacción una acción automática en una situación anteriormente varias veces realizada. Esta intuición está basada en una imagen de un escenario de pérdida del 30 al $40 \%$ del capital (basada en cálculos), que produce una anticipación frente al resultado de ese negocio, y se piensa que tal resultado será semejante al previsto, por eso es tan sorprendente cuando no sucede así. En este caso, la semejanza no ocurre y se genera un efecto de sorpresa por contraste. También, la decisión se basa en la similitud de experiencias anteriores con la actual, en las cuales ha confiado en el sujeto $B$, y han sido exitosas, lo cual no curre en esta nueva situación.

Desde el aspecto antropológico, es posible conjeturar que el "bombardeo de significantes" contribuye a que el decisor asuma que la información que recibe de su asistente es correcta, evitando leer el contrato y hacer un doble chequeo. El problema no es necesariamente que se base en una lógica imaginaria, sino que se base en prejuicios falsos (juicios no analizados). No se evidencia claramente aquí el aspecto psicológico en los términos definidos por Malvezzi, pero puede abordarse a continuación desde la perspectiva de los aspectos lógicos de la intuición.

Al respecto, el entrevistado afirma que aunque teóricamente la decisión de comprar determinadas acciones es acertada, la emocionalidad juega un papel importante que puede conducir a errores "por la cantidad de actores del mercado, porque hay gente que en la 
racionalidad piensa que ese dato que salió es distinto y se puede interpretar de una manera indiferente". Y agrega que se trata de una "decisión emocional y a la vez racional [...]. Es pensada, pero llega un momento en que la parte racional no permite ningún análisis distinto a 'voy a barrer ya de primero', y eso lleva muchas veces a errores".

El entrevistado introduce así otros dos factores en este tipo de decisiones en contextos de un mundo globalizado, además de la velocidad y la emocionalidad, se trata la competitividad y de la interpretación. En efecto, aunque Malvezzi no to expresa explícitamente, es posible colegir de lo que plantea el entrevistado que la globalización, al generar una interacción mayor entre diferentes actores sociales, genera una competitividad muy alta, y diferentes posibilidades de interpretación de la información. Todos estos elementos contribuyen a la incertidumbre de la situación, esto es, a un grado de probabilidad de acierto que no se calculó ni predijo acertadamente. Además, los prejuicios, si bien son de los sujetos, están claramente asociados al contexto de globalización.

Entonces, la velocidad de la decisión, asociada a la alta competitividad y a la alta emocionalidad, expresa el contexto de la interpretación que conforma la decisión. La decisión rápida se asocia usualmente a la lógica primaria, porque a mayor velocidad menor posibilidad de usar la lógica secundaria, que implica tiempo para deliberar, esto es, detenerse a considerar los pros y contras de una situación. Asimismo, la toma de decisiones en contextos de incertidumbre se asocia a la respuesta intuitiva, pues cuando no es posible calcular el grado de riesgo con métodos probabilísticos formalizados, el uso de la intuición tiende a ser mayor; no obstante, es importante tener presente que en el 'mundo real', en la vida cotidiana, nunca se da una decisión basada únicamente en la lógica secundaria (Gigerenzer, 2008).

En el caso narrado encontramos, entonces, una mezcla o alternancia de aspectos de lógica primaria y lógica secundaria. La lógica primaria se evidencia cuando el entrevistado afirma, racionalizando, que la culpa fue de él, sujeto A, y no del sujeto B, porque no leyó el contrato. Otra forma de decirlo es que su decisión fue no-consciente, y no utilizó en ese momento la lógica secundaria, sino que se basó en la sensación de confianza imaginaria que tenía en el sujeto $B$, aun sabiendo que era una persona muy joven $y$, por consiguiente, con poca experiencia. Entonces, como conjetura podemos pensar que asoció inconscientemente la sensación de confianza con la sensación de estar haciendo lo adecuado y sintió mucha tranquilidad al delegar la tarea (reconocemos aquí el mecanismo de contigüidad). En esta situación, el sujeto A captó indicios que no fueron escuchados a tiempo, lo cual asociamos con un privilegio de la lógica imaginaria sobre la lógica simbólica. Si hubiera utilizado en ese momento la lógica secundaria, basada en la no-contradicción, habría pensado algo como: dado que el sujeto $B$ es muy joven, y dado que la juventud implica falta de experiencia y que la falta de experiencia hace más proclive al sujeto a 
cometer errores, el sujeto B es proclive a cometer errores y debo revisar atentamente lo que hace; pero no fue este el caso. Además, podemos entender que el sujeto A se dejó guiar por el principio de placer, que está asociado a los resultados inmediatos de evitar el displacer que implicaría leer el contrato en su totalidad y detenidamente, quizás por evitar 'perder tiempo'; en cambio optó por confiar en lo que le dijo el sujeto B. Lo paradójico es que por evitar un displacer inmediato, se genera un displacer mayor a mediano plazo. En términos de Kahneman (2012), se acudió a la economía cognitiva propia del sistema 1, basada en la aversión a la pérdida.

Además, podemos encontrar el mecanismo de semejanza cuando el sujeto B asume que lo que escribió por correo al sujeto A "correspondía a lo que estaba escrito en el contrato". Al parecer, lo encontró tan semejante que le pareció igual. Si el sujeto B hubiera utilizado la lógica secundaria, habría analizado más detenidamente la semejanza aparente, intentando captar las diferencias entre lo escrito en el correo y en el contrato.

Luego, el sujeto B llega "sudando", como manifestación de las emociones (en su componente fisiológico) que lo invadieron en el momento en que se dio cuenta de su error de interpretación, y siente en el cuerpo el malestar propio que genera la equivocación y, podemos conjeturar, el temor a las consecuencias. Aquí puede conjeturarse que esta experiencia dejó una marca somática, en el sentido de Damasio (2006), en el sujeto B. Pero también en el sujeto A, quien lo expresa más claramente cuando afirma que sintió "mucha rabia, mucha impotencia, me sentí muy mal conmigo mismo". En este punto estaba experimentado sensaciones y emociones asociadas a la situación, y que lo llevaron a un aprendizaje que comprendió con el cuerpo: aprendió que no debía delegar "con tanta tranquilidad", esto es, aprendió a analizar la situación conscientemente, hacer un "doble chequeo", aunque su sensación fuera de tranquilidad, especialmente cuando se trata de asuntos que para él son tan delicados como es el caso del dinero. Aquí encontramos cómo ese aprendizaje, producto de un error que desencadenó una serie de emociones que funcionan sobre la base de la lógica primaria, condujo a un aprendizaje que expresa en términos de lógica secundaria.

Con relación a la articulación o mezcla de la lógica primaria y secundaria, es posible pensar que cuando se comete un error o hay una contradicción (propia de la lógica secundaria) se produce una reacción física, tal y como lo ha planteado el neurólogo Antonio Damasio (2006), y si se es consciente se experimenta cierto grado de malestar. Ramírez (2011, Cav. 84) sugiere que Freud, como neurólogo, al parecer tenía algo de razón al considerar que el cerebro 'traduce' la contradicción en 'términos' de sus leyes fisiológicas, lo cual está siendo probado por estudios contemporáneos. 
Otro aspecto que es importante mencionar, aunque no es posible analizar aquí debido a la poca información que tenemos y a los límites de este texto, es el papel inconsciente de los dos errores cometidos en el caso: el del sujeto A (delegar en exceso) y el del sujeto B (interpretar mal los datos). En términos generales, desde una perspectiva psicoanalítica, los errores tienen un sentido inconsciente que, para el sujeto, es importante analizar y hacer consciente, con el fin de conocer su proyecto existencial y qué aspectos lo obstaculizan. De esta manera, el sujeto puede captar una serie de prejuicios, esto es, creencias, valores que determinan su actuar, pero que operan como dogmas porque nunca los ha cuestionado. Además, al hacerlo, poco a poco asume de manera más consciente la responsabilidad que tiene sobre los efectos de las decisiones y las elecciones que realiza.

\section{A manera de conclusión}

En el mundo globalizado en que nos tocó vivir, en el que la economía de mercado tiene tanta relevancia, muchas veces se deja de lado el aspecto ético, el examen de los propios valores, para determinar su conveniencia respecto a sí mismo y a los otros. En un contexto en el que la productividad es el paradigma de progreso y la competitividad es un medio para lograrlo, hablar de una ascesis subjetiva, de una revisión de la propia vida en la que se tenga en cuenta a los otros seres, se constituye en un asunto fundamental para estar a la altura que el desarrollo de la tecnología nos presenta desde una perspectiva ética. Según Konrad Lorenz (1993) el avance de la tecnología es más veloz que el avance ético de la humanidad, y es este el principal reto que tenemos como especie para evitar la autodestrucción. En este sentido, es representativa la serie Black mirror, de Netflix, que expone desde una perspectiva distópica, las ventajas y peligros del desarrollo de la tecnología en un mundo globalizado.

Con estas reflexiones, no estamos cuestionando el caso que presentamos; este simplemente nos sirve de referente para mostrar las posibilidades que nuestra propuesta abre con relación al análisis de la toma de decisiones. El análisis que iniciamos nos lleva a considerar el contexto en el que se toman las decisiones, y algunos aspectos del proceso decisorio. Pero lo más importante es que permite captar que al considerar los aspectos lógicos de la intuición, se abren caminos de análisis que involucran tanto el contexto como los aspectos subjetivos del decisor.

Este tipo de análisis puede aplicarse a las decisiones más importantes que son las decisiones y elecciones existenciales, las que tomamos cotidianamente pero que tienen repercusiones sobre todo nuestro proyecto de vida. Inclusive, planteamos que toda decisión puede tener 
consecuencias existenciales. Por ejemplo, pensemos en lo que implica tomar una decisión aparentemente trivial como empezar a salir con alguien. Al respecto, Erich Fromm (1985) plantea cómo muchas elecciones implican que cada vez se cierra más el margen de libertad y la posibilidad de revertir la decisión. Propone, entonces, el ejemplo de un niño de 8 años que tiene un amigo que a la mamá no le gusta por causa de su origen, por lo que le dice que si deja de frecuentarlo lo llevará al circo. El niño accede a la presión de la madre, acto que Fromm (1985) califica como una traición a sí mismo. Las consecuencias son: sentirse avergonzado, perder el sentimiento de integridad, así como la confianza en símismo. Diez años después, se enamora de una joven, es un sentimiento muy fuerte, piensan formalizar el compromiso, pero ella es de un origen que a sus padres les disgusta. Intentan disuadirlo, pero él se mantiene firme; entonces le prometen un viaje a Europa si acepta aplazar la formalización del compromiso. Él acepta, después de todo, qué importa esperar unos meses más, además el viaje será conveniente y no disminuirá el amor por ella. Pero en el viaje conoce a otras jóvenes, su vanidad se siente satisfecha y, antes de regresar, le escribe a su novia para terminar su relación. ¿̇Cuándo tomó la decisión?, pregunta Fromm. No fue cuando escribió la carta, dice, sino cuando eligió ir al viaje a Europa. Inconscientemente sintió que se había vendido, y tuvo que romper la relación. Se traicionó de nuevo, ahondando la debilidad interior y la falta de confianza en sí mismo, aunque ocultas tras sus nuevas conquistas. Termina entrando en el negocio de su padre, y no estudia física, carrera que le gusta y para lo cual tiene vocación. Se casa con la hija de unos amigos ricos de su padre, se convierte en un hombre de negocios y un político influyente que toma decisiones contra la voz de su propia conciencia porque teme enfrentarse a la opinión pública. Cada vez más su posibilidad de decidir se hizo más estrecha. Así, dice Fromm, terminan muchas personas restringiendo su libertad, como en el caso de empleados de Hitler o Stalin. Lo contrario, dice, también es cierto: la primera decisión acertada, hace más fácil la siguiente decisión acertada, porque la persona se acostumbra a captar dónde hay una bifurcación en la cual aún tiene opciones para elegir lo correcto de acuerdo con su propio ser.

Podemos tomar otro ejemplo de la serie de Netflix, Breaking bad. Cada decisión que toma el protagonista va haciendo más estrecho su margen de libertad y más difícil su posibilidad de redimirse. Cada uno de los pequeños pasos que da en el mundo del tráfico de drogas, va marcando su trayectoria, determinado sus elecciones futuras, hasta que llega al punto de no retorno.

En el caso presentado quedan varias preguntas, como por ejemplo: $\dot{\imath} e n$ qué momento tomaron la decisión realmente los sujetos $A$ y B? ¿̇Qué motivó en cada uno la decisión? ¿̇e qué manera influyó su sistema de valores no analizados (pre-juicios) en las equivocaciones? ¿̇Quisieran 
saber más acerca de los mecanismos que están en juego en esa decisión? Son preguntas que les corresponde resolver a ellos, si así lo deciden.

Por nuestra parte, en resumen, proponemos que toda decisión en la vida cotidiana está basada en la lógica primaria y la lógica secundaria. Las intuiciones permiten que cada sujeto conozca un poco más de sí, de sus valores, pre-jucios, pre-supuestos, creencias. Por tal motivo es importante analizar las intuiciones, para identificar cuál fue la lógica de tal elección, con el fin de comprender en qué pre-juicios o pre-supuesto se basó. Si aceptamos lo anterior, aceptaremos que esta perspectiva de análisis en términos de los aspectos lógicos de la intuición, que presentamos brevemente, puede ser de utilidad para analizar las decisiones en diferentes ámbitos, bien sea para determinar si hubo errores en la toma de decisiones evaluando el proceso y los resultados, bien sea para contribuir a la formación de la intuición y la toma de decisiones, con el fin de aprender de los errores y de los aciertos, generando así en cada participante un saber sedimentado, depurado de prejuicios, que contribuya a mejorar la validez en cuanto a la toma de decisiones y las elecciones, especialmente las elecciones existenciales y éticas (que son más de las que pensamos). Pero para ponerla en práctica, lo óptimo es que las personas implicadas en el proceso decisorio realicen el análisis, con el fin de que se genere una transformación en ellas que repercuta en su forma de tomar decisiones.

\section{Referencias bibliográficas}

Autor y Autor (2017).

Autor (2017).

Damasio, A. (2006). El error de Descartes: la emoción, la razón y el cerebro humano. Barcelona: Editorial Crítica.

Dane, E. \& Pratt, M.G. (2007). Exploring intuition and its role in managerial decision making. Academy of Management Review, 32(1), 33-54. doi:10.5465/AMR.2007.23463682

Freud, S. (1912/2010). Nota sobre el concepto de lo inconsciente en psicoanálisis (1912). En: Obras completas, vol. 12. Buenos Aires: Amorrortu, pp. 265-277.

Fromm, E. (1985). El corazón del hombre. México: Fondo de Cultura Económica. 
Gigerenzer, G. (2008). Decisiones instintivas. La inteligencia del inconsciente. Barcelona: Ariel.

Kahneman, D. (2012). Pensar rápido, pensar despacio. Barcelona: Debate.

Lévi-Strauss, C. (1995). La eficacia simbólica. En: Antropología estructural (pp. 2011-227). Barcelona: Paidós.

Lopera, J.; Echeverri, J. y Goenaga, J. (en prensa). De la sabiduría práctica y la toma de decisiones en incertidumbre. Informe de investigación. Universidád de Antioquia.

Lopera, J.; Ramírez, C.; Zuluaga, M. y Ortiz, J. (2010). El método analítico como método natural. Nómadas. Critical Journal of Social and Juridical Sciences, 25(1). http://www.redalyc.org/pdf/181/18112179017.pdf

Lorenz, K. (1993). Sobre la posibilidad de una síntesis entre las ciencias de la naturaleza y las ciencias del espíritu. En: La ciencia natural del hombre. "El manuscrito de Rusia" (1944-1948) (pp. 102-132). Barcelona: Tusquets.

Malvezzi, S. (2016). Psicología organizacional y del trabajo. De la administración científica a la globalización: una historia de desafíos (pp. 27-42). En: Orejuela, Johnny; Andrade, Verónica y Villamizar, Milena (Editores). Psicología de las organizaciones y del trabajo. Apuestas de investigación II. Cali: Editorial Bonaventuriana.

Ramírez, C. (2012). La vida como un juego existencial: Ensayitos. Medellín: Fondo Editorial Universidad EAFIT.

Ramírez, C. (2011). Cav. 84. Contradicción. En: Apuntes. Medellín: Texto policopiado por el grupo de investigación el Método analítico. Universidad de Antioquia.

Ramírez, C.; Lopera, J.; Zuluaga, M. y Ortiz, J. (2017). El método analítico. Bogotá: San Pablo.

Ricoeur, P. (1950/1986). El proyecto y la motivación. Lo voluntario y lo involuntario, vol. I. Buenos Aires: Docencia.

Sadler-Smith, E. (2016). 'What happens when you intuit?': Understanding human resource practitioners' subjective experience of intuition through a novel linguistic method. Human Relations, 69(5), 1069-1093. doi:10.1177/0018726715602047 